ESAIM: COCV 18 (2012) 969-986

DOI: $10.1051 / \mathrm{cocv} / 2011198$
ESAIM: Control, Optimisation and Calculus of Variations

www.esaim-cocv.org

\title{
NASH EQUILIBRIA FOR A MODEL OF TRAFFIC FLOW WITH SEVERAL GROUPS OF DRIVERS
}

\author{
Alberto Bressan ${ }^{1}$ And Ke Han ${ }^{1}$
}

\begin{abstract}
Traffic flow is modeled by a conservation law describing the density of cars. It is assumed that each driver chooses his own departure time in order to minimize the sum of a departure and an arrival cost. There are $N$ groups of drivers, The $i$-th group consists of $\kappa_{i}$ drivers, sharing the same departure and arrival costs $\varphi_{i}(t), \psi_{i}(t)$. For any given population sizes $\kappa_{1}, \ldots, \kappa_{n}$, we prove the existence of a Nash equilibrium solution, where no driver can lower his own total cost by choosing a different departure time. The possible non-uniqueness, and a characterization of this Nash equilibrium solution, are also discussed.
\end{abstract}

Mathematics Subject Classification. 35E15, 49K20, 91A12.

Received August 20, 2011.

Published online 16 January 2012.

\section{INTRODUCTION}

Consider a model of traffic flow where $n$ groups of drivers travel along a freeway, from a location $A$ (say, a residential neighborhood) to a location $B$ (the city center). Each driver seeks to optimize his departure time, given a cost for starting early, and a cost for arriving late. Different groups have different departure and arrival costs. For example, there may be three groups: drivers in the first group need to arrive at destination by 8:00 am., those in the second group need to arrive by 9:00 am., while drivers in the third group can arrive at any time between 9:00 and 12:00, and simply wish to minimize the time spent on the road.

Following the classical papers [13,14], traffic flow will be modeled by the conservation law

$$
\rho_{t}+[\rho v(\rho)]_{x}=0 .
$$

Here $\rho=\rho(t, x)$ is the density of cars, while $v(\rho)$ is their speed. In our model, a basic assumption is that drivers of different groups are distinguished only by their costs, not by the vehicles they drive. In other words, the speed $v(\cdot)$ as a function of traffic density is the same for all groups. Calling $\rho_{i}=\rho_{i}(t, x)$ the density of drivers of the $i$-th group, the scalar function $\rho=\rho_{1}+\cdots+\rho_{n}$ provides an entropy solution to the conservation law (1.1).

Aim of this paper is to study Nash equilibria. These are solutions where no single driver can lower his own cost by changing his own departure time. In the case $n=1$, where all drivers share the same cost, the existence, uniqueness, and a characterization of Nash solutions were recently established in [2].

\footnotetext{
Keywords and phrases. Scalar conservation law, Hamilton-Jacobi equation, Nash equilibrium.

1 Department of Mathematics, Penn State University University Park, 16802 Pa, USA. bressan@math.psu.edu; kxh323@psu.edu
} 
The main features of the general case $n \geq 2$, and the new results proved in the present paper, can be summarized as follows.

- A solution to the traffic flow problem with $n$ groups of drivers can be determined by assigning a scalar density $\rho=\rho(t, x)$, satisfying the conservation law (1.1), together with a "prioritizing function". Among the first $\beta$ drivers who have departed within a given time $t$, this function specifies how many belong to the first group, how many to the second group, etc.;

- for any $n$-tuple of costs $c=\left(c_{1}, \ldots, c_{n}\right)$, there exists at least one Nash equilibrium solution where all drivers of the $i$-th group share the same total cost $c_{i}$. The corresponding density function $\rho$ in (1.1) is uniquely determined by $c$. However, for the same $n$-tuple of costs $\left(c_{1}, \ldots, c_{n}\right)$, there can be several different decompositions $\rho=\rho_{1}+\cdots+\rho_{n}$, yielding different Nash equilibria. In general, the map

$$
\begin{aligned}
c \mapsto \kappa(c) \doteq\left\{\left(\kappa_{1}, \ldots, \kappa_{n}\right) ; \quad \kappa_{i} \doteq \int \rho_{i}(t, x) \mathrm{d} x \quad\right. \text { is the the total number } \\
\text { of } \left.i \text {-drivers in some Nash solution with costs }\left(c_{1}, \ldots, c_{n}\right)\right\}
\end{aligned}
$$

is a multivalued function. On the other hand, the map $c \mapsto \kappa_{1}+\cdots+\kappa_{n}$ is always single valued;

- the multifunction $c \mapsto \kappa(c) \subset \mathbb{R}^{n}$ in (1.2) has closed graph and nonempty, compact, convex values. Relying on Cellina's approximate selection theorem [3], a topological argument shows that $\bigcup_{c \in \mathbb{R}^{n}} \kappa(c)=\mathbb{R}_{+}^{n}$. Otherwise stated, for every $n$-tuple $\left(\kappa_{1}, \ldots, \kappa_{n}\right)$ with $\kappa_{i} \geq 0$ for each $i$, there exists a vector $c=\left(c_{1}, \ldots, c_{n}\right)$ of costs and a Nash equilibrium solution where the total number of drivers of the $i$-th group is $\kappa_{i}$ and each $i$-driver pays the same total $\operatorname{cost} c_{i}$. When $n \geq 2$ this solution may not be unique.

In our model, $x \in[0, R]$ is the space variable, describing a point along the road. A solution of (1.1) is determined by assigning the incoming flow

$$
\rho(0, t) v(\rho(0, t))=\bar{u}(t)
$$

at the entrance of the freeway. As remarked in [2], it is more convenient to switch the roles of the independent variables $t, x$, and rewrite the boundary value problem (1.1), (1.3) as a Cauchy problem for the flux of cars $u=\rho v(\rho)$, namely

$$
u_{x}+f(u)_{t}=0, \quad u(t, 0)=\bar{u}(t) .
$$

Here $u \mapsto f(u)=\rho$ is defined as a partial inverse of the map $\rho \mapsto \rho v(\rho)=u$, as in Figure 1.

The remainder of the paper is organized as follows. In Section 2 we clarify all the assumptions and state the main result. Section 3 is devoted to the analysis of Nash equilibria for a given vector of costs $\left(c_{1}, \ldots, c_{n}\right)$, while in Section 4 we prove the existence of an equilibrium solution for given population sizes $\left(\kappa_{1}, \ldots, \kappa_{n}\right)$.

For a general introduction to scalar conservation laws and the Lax formula we refer to [11,12,15]. A short introduction to control theory and Hamilton-Jacobi equations can be found in [4]. All the basic results on setvalued functions, including Cellina's approximate selection theorem, can be found in [1]. Various optimization problems for traffic flow, based on the Lighthill-Whitham conservation law model, have been considered in [7-10]. For an introduction to differential games and for recent applications to traffic flow on networks we refer to [5] and [6], respectively.

\section{Statement of the MAIn ReSUlts}

Consider the conservation law

$$
u_{t}+f(u)_{x}=0 \quad(t, x) \in[0, T] .
$$

In our traffic model, after interchanging the roles of the variables, $x$ will denote time while $t \in[0, T]$ describes points along a highway of total length $T$. The dependent variable $u=u(t, x)$ denotes the traffic flow at time 

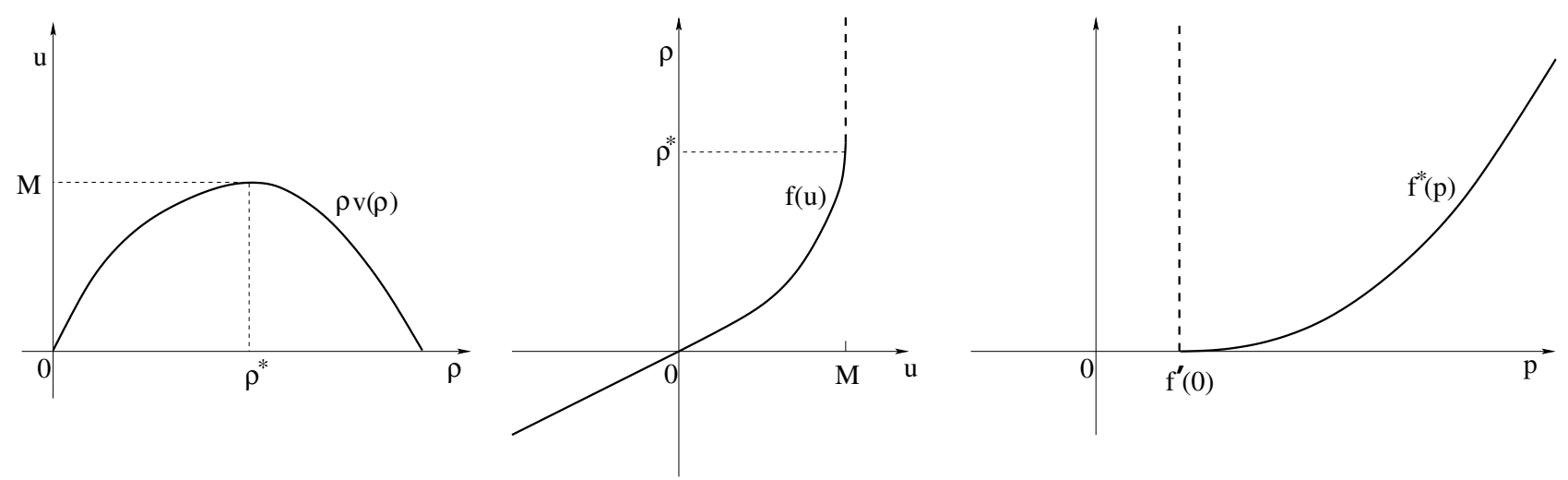

FIGURE 1. Left: the function $\rho \mapsto \rho v(\rho)$ describing the flux of cars. Middle: the function $f$, implicitly defined by $f(\rho v(\rho))=\rho$ and extended according to (2.2). Right: the Legendre transform $f^{*}$.

$x$ across the point $t$ on the highway. In the Lighthill-Whitham model one has $u=\rho v(\rho)$, where $\rho$ is the density of cars while $v(\rho)$ is the speed of cars (see Fig. 1). The function $u \mapsto f(u)=\rho$ is obtained by inverting the function $\rho \mapsto \rho v(\rho)=u$, over the interval $\left[0, \rho^{*}\right]$ where $\frac{\partial}{\partial \rho}[\rho v(\rho)] \geq 0$. As in [2], we extend $f$ to a function $f: \mathbb{R} \mapsto \mathbb{R} \cup\{+\infty\}$, by setting (see Fig. 1)

$$
f(u) \doteq \begin{cases}f^{\prime}(0+) u & \text { if } u<0 \\ +\infty & \text { if } u>M\end{cases}
$$

Introducing the integral function

$$
U(t, x) \doteq \int_{-\infty}^{x} u(t, s) \mathrm{d} s,
$$

the conservation law (2.1) can be equivalently written as a Hamilton-Jacobi equation

$$
U_{t}+f\left(U_{x}\right)=0
$$

Throughout the following, $f$ denotes the flux function extended to the entire real line as in $(2.2)$, while

$$
f^{*}(p) \doteq \max _{u}\{p u-f(u)\}
$$

is the Legendre transform of $f$. In our model, $U(t, x)$ describes the total number of cars that have crossed the point $t$ along the highway during the time interval $]-\infty, x]$.

As initial data we shall consider any non-decreasing, function $Q: \mathbb{R} \mapsto \mathbb{R}^{+}$, with

$$
Q(-\infty)=0, \quad Q(+\infty)=\kappa<+\infty .
$$

Here $Q(x)$ describes the total number of drivers that have initiated their journey (possibly joining a queue at the entrance of the highway, if there is any) at time $\leq x$.

Notice that $Q$ is continuous except for countably many times $x$. To fix the ideas, we shall consider the leftcontinuous version where $Q(x)=Q(x-)$ coincides with its left limit at every $x$. When needed, we shall denote by $Q(x+)=\lim _{y \rightarrow x+} Q(y)$ the right limit of $Q$ at $x$.

For a given $Q(\cdot)$, consider the Lipschitz continuous function

$$
\bar{U}(x) \doteq \inf \{Q(y)+M(x-y) ; y \leq x\} \leq Q(x) .
$$


The difference $Q(x)-\bar{U}(x)$ can be interpreted as the length of the queue at the entrance of the highway at time $x$, while $\bar{U}(x)$ denotes the total number of drivers that have actually departed (after clearing the queue) up to time $x$.

For $t>0$, the entropy-admissible solution to the Cauchy problem (2.4), (2.7) is provided by the Lax formula:

$$
U(t, x)=\min \left\{t f^{*}\left(\frac{x-y}{t}\right)+\bar{U}(y) ; \quad y \in \mathbb{R}\right\}=\min \left\{t f^{*}\left(\frac{x-y}{t}\right)+Q(y) ; \quad y \in \mathbb{R}\right\} .
$$

Observe that the last two expressions in (2.8) are equal because $\left(f^{*}\right)^{\prime}(p) \leq M$ for all $p$, and $\left(f^{*}\right)^{\prime}(p) \rightarrow M$ as $p \rightarrow+\infty$. Moreover, $U(0+, x)=\bar{U}(x)$.

As in [2], we consider a cost $\varphi(x)$ for early departure and a cost $\psi(x)$ for late arrival. The basic assumptions will be:

(A1) The flux function $f:[0, M] \mapsto \mathbb{R}$ is continuous. Moreover, it is twice continuously differentiable on the open interval $] 0, M[$ and satisfies $f(0)=0$, together with

$$
f^{\prime}(u) \geq b_{1}>0, \quad f^{\prime \prime}(u) \geq b_{2}>0 \quad \text { for } 0<u<M, \quad \lim _{u \rightarrow M-} f^{\prime}(u)=+\infty .
$$

(A2) The cost functionals $\varphi, \psi$ are locally Lipschitz continuous and satisfy

$$
\left\{\begin{array} { l } 
{ \varphi ^ { \prime } ( x ) \leq - \delta _ { 0 } < 0 , } \\
{ \psi ( x ) \geq 0 , \quad \psi ^ { \prime } ( x ) \geq 0 , }
\end{array} \quad \left\{\begin{array}{l}
\lim _{x \rightarrow-\infty} \varphi(x)=+\infty \\
\lim _{x \rightarrow+\infty}(\varphi(x)+\psi(x))=+\infty
\end{array}\right.\right.
$$

According to (A2), the cost for early departure is decreasing in time, while cost for late arrival is increasing. The assumption that these costs tend to infinity as $t \rightarrow \pm \infty$ coincides with common sense and guarantees that in an equilibrium solution the departure rate is compactly supported.

Given an initial data $Q(\cdot)$ as in $(2.6)$, for $\beta \in\left[0, \kappa\left[\right.\right.$ we define the points $x^{q}(\beta), x^{d}(\beta)$, and $x^{a}(\beta)$ by setting

$$
\left\{\begin{array}{l}
x^{q}(\beta)=\max \{x \in \mathbb{R} ; \quad Q(x) \leq \beta\}, \\
x^{d}(\beta)=\max \{x \in \mathbb{R} ; \quad U(0+, x) \leq \beta\}, \\
x^{a}(\beta)=\max \{x \in \mathbb{R} ; \quad U(T, x) \leq \beta\} .
\end{array}\right.
$$

In the application to traffic flow, $\beta$ is a Lagrangian variable labeling a particular driver. In this case, $x^{q}(\beta)$ accounts for the time where this driver joins the queue, $x^{d}(\beta)$ is the actual departure time and $x^{a}(\beta)$ is the arrival time. We observe that, for all except countably many $\beta$, the points $x^{q}(\beta)$ and $x^{a}(\beta)$ are uniquely determined by the relations

$$
Q\left(x^{q}(\beta)\right) \leq \beta \leq Q\left(x^{q}(\beta)+\right), \quad U\left(T, x^{a}(\beta)\right)=\beta .
$$

It will be convenient to introduce the function

$$
h(s) \doteq-T f^{*}\left(-\frac{s}{T}\right),
$$

where $f^{*}$ is the Legendre transform of $f$, as in (2.5). For a.e. $\beta$ the arrival time $x^{a}(\beta)$ can also be characterized as

$$
x^{a}(\beta)=\inf \{x ; \quad Q(y) \geq \beta+h(y-x) \text { for all } y \leq x\} .
$$

Defining the constant

$$
\mu=\frac{[\text { length of the highway }]}{[\text { maximum speed }]}=T f^{\prime}(0),
$$


for a driver that departs at an arbitrary time $x$ we define the arrival time as

$$
A(x) \doteq \max \left\{x+\mu, \sup _{\beta<Q(x)} x^{a}(\beta)\right\} .
$$

According to (2.16), if there is no traffic at all, then the total time needed for the trip is $\mu$. On the other hand, if the driver starting at time $x$ encounters traffic, his arrival time will be the supremum among the arrival times of all cars departed earlier.

The following definition was introduced in [2].

Definition 2.1. We say that a bounded, nondecreasing initial data $Q(\cdot)$ satisfying $(2.6)$ yields a Nash solution of the Cauchy problem (2.4)-(2.7) with initial and terminal cost functions $\varphi, \psi$ if there exists a constant $c$ such that:

(i) for almost every $\beta \in[0, \kappa]$ one has

(ii) for all $x \in \mathbb{R}$, there holds

$$
\varphi\left(x^{q}(\beta)\right)+\psi\left(x^{a}(\beta)\right)=c
$$

$$
\varphi(x)+\psi(A(x)) \geq c .
$$

In connection with the traffic model, condition (i) states that all drivers bear the same cost $c$. Condition (ii) says that, regardless of the starting time $x$, no one can achieve a cost $<c$.

Next, assume that there are different groups of drivers that need to reach destination at different times. For $i=1, \ldots, n$ we thus consider cost functions $\varphi_{i}(x), \psi_{i}(x)$, all satisfying the assumptions (A2). We seek Nash equilibrium solutions in this more general situation.

Let $Q_{1}(\cdot), \ldots, Q_{n}(\cdot)$ describe the departure distributions of the various groups of drivers. In other words, $Q_{i}(x)$ is the total number of drivers of the $i$-th group that have started their journey (possibly joining the queue at the entrance of the highway) within time $x$. We assume that all these functions are left continuous and non-decreasing, with

$$
Q_{i}(-\infty)=0, \quad Q_{i}(+\infty)=\kappa_{i} .
$$

For $i=1, \ldots, n$, the value $\kappa_{i} \geq 0$ accounts for the total number of drivers of the $i$-th group.

We will define a solution $\left(U_{1}, \ldots, U_{n}\right)(t, x)$ to the traffic flow problem, corresponding to the above departure pattern. Here $U_{i}(t, x)$ describes the total number of drivers of the $i$-th group that have crossed the point $t$ on the highway within time $x$. Setting

$$
Q(x) \doteq \sum_{i=1}^{n} Q_{i}(x), \quad U(t, x) \doteq \sum_{i=1}^{n} U_{i}(t, x),
$$

it is clear that the function $U$ should provide an admissible solution to equation (2.4) with initial data $Q(\cdot)$. This implies that the function $U$ is uniquely determined by the Lax formula (2.8). In general, however, that this is not enough to uniquely determine the components $U_{i}$. For example, if $Q_{i}$ and $Q_{j}$ both have a jump at $x=x_{0}$, this means that at the same instant of time $x_{0}$ a positive amount of drivers join the queue, both from the $i$-th and from the $j$-th group. Different solutions can then be obtained, depending on the priority that we assign to drivers in each group. To resolve this issue, we consider a family of nondecreasing functions $\mathcal{B}_{i}:[0, \kappa] \mapsto\left[0, \kappa_{i}\right]$ with the following properties:

$$
\begin{array}{cc}
\sum_{i=1}^{n} \mathcal{B}_{i}(\beta)=\beta & \text { for all } \beta \in[0, \kappa], \\
\mathcal{B}_{i}(Q(x))=Q_{i}(x) & \text { for all } x \in \mathbb{R}, \quad i \in\{1, \ldots, n\} .
\end{array}
$$


Remark 2.2. Given the left continuous functions $Q_{1}, \ldots, Q_{n}$ as in (2.19), an $n$-tuple of functions $\mathcal{B}_{i}$ satisfying the above properties can be constricted as follows. Consider the left-continuous, nondecreasing function $x \mapsto Q(x) \doteq \sum_{i} Q_{i}(x) \in[0, \kappa]$. If $\beta=Q(x)$ for some $x$, we then define

$$
\mathcal{B}_{i}(\beta)=Q_{i}(x) .
$$

On the other hand, if $Q(x)<\beta \leq Q(x+)$, we define

$$
\mathcal{B}_{i}(\beta) \doteq Q_{i}(x)+\frac{\beta-Q(x)}{Q(x+)-Q(x)}\left[Q_{i}(x+)-Q_{i}(x)\right] .
$$

It is now easy to check that both conditions (2.21)-(2.22) are satisfied.

Given an initial data $Q(\cdot)$ as in $(2.6)$ and the prioritizing functions $\mathcal{B}_{i}:[0, \kappa] \mapsto\left[0, \kappa_{i}\right]$, the functions $U_{i}$ in $(2.20)$ are now uniquely determined by the formulas

$$
U_{i}(t, x)=\mathcal{B}_{i}(U(t, x)) .
$$

Here, for $t>0$,

$$
U(t, x)=\min \left\{t f^{*}\left(\frac{x-y}{t}\right)+Q(y) ; \quad y \in \mathbb{R}\right\}
$$

is the solution of (2.4) with initial data $Q(\cdot)$, computed by the Lax formula.

We wish to introduce a concept of Nash equilibrium solution, extending Definition 2.1 to the case of several groups of drivers with different costs. Toward this goal, we first need to determine the starting time (possibly joining the queue at the entrance of the highway) and the arrival time of each driver in the $i$-th group. Let $\zeta \in\left[0, \kappa_{i}\right]$ label this particular driver. We then define

$$
\begin{cases}x_{i}^{q}(\zeta)=\max \{x \in \mathbb{R} ; & \left.Q_{i}(x) \leq \zeta\right\}, \\ x_{i}^{a}(\zeta)=\max \{x \in \mathbb{R} ; & \left.U_{i}(T, x) \leq \zeta\right\} .\end{cases}
$$

Notice that, for all but countably many $\zeta$, these values are uniquely determined by the relations

$$
Q_{i}\left(x_{i}^{q}(\zeta)\right) \leq \zeta \leq Q_{i}\left(x_{i}^{q}(\zeta)+\right), \quad U_{i}\left(T, x_{i}^{a}(\zeta)\right)=\zeta .
$$

Definition 2.3. Consider a left continuous, nondecreasing initial data $Q(\cdot)$ as in $(2.6)$, and let $\mathcal{B}_{i}:[0, \kappa] \mapsto\left[0, \kappa_{i}\right]$ be non-decreasing, surjective maps satisfying (2.21).

We say that the starting distribution $Q(\cdot)$ together with the prioritizing functions $\mathcal{B}_{1}, \ldots, \mathcal{B}_{n}$ yield a Nash equilibrium solution to the traffic flow problem with cost functions $\varphi_{i}, \psi_{i}$ if there exist constants $c_{1}, \ldots, c_{n}$ such that, for $i=1, \ldots, n$, the following holds.

(i) For almost every $\zeta \in\left[0, \kappa_{i}\right]$ one has

$$
\varphi_{i}\left(x_{i}^{q}(\zeta)\right)+\psi_{i}\left(x_{i}^{a}(\zeta)\right)=c_{i}
$$

(ii) for all $x \in \mathbb{R}$, one has

$$
\varphi_{i}(x)+\psi_{i}(A(x)) \geq c_{i}
$$

Here $x \mapsto A(x)$ is the function in (2.16), describing the arrival time of a driver starting at time $x$. According to (i), every driver of the $i$-th group pays the same $\operatorname{cost} c_{i}$, while by (ii) he cannot decrease this cost by choosing any other starting time. We can now state our main result of existence of Nash equilibria.

Theorem 2.4. Let the flux function $f$ satisfy (A1), and let the cost functions $\varphi_{i}, \psi_{i}$ for the various groups of drivers satisfy (A2), for every $i \in\{1, \ldots, n\}$. Then, for every $n$-tuple $\left(\kappa_{1}, \ldots, \kappa_{n}\right)$ of non-negative numbers there exists a Nash equilibrium solution, where $\kappa_{i}$ is the total number of drivers of the $i$-th group. 


\section{NASH EQUilibria WITH GIVEN COSTS}

As a first step toward the proof of Theorem 2.4, given constants $c_{1}, \ldots, c_{n}$, in this section we construct a Nash equilibrium solution where all drivers of the $i$-th group pay the same cost $c_{i}$.

Let $x \mapsto \Lambda_{i}(x)$ be the map implicitly defined by

$$
\varphi_{i}\left(\Lambda_{i}(x)\right)+\psi_{i}(x)=c_{i} .
$$

In other words, if an $i$-driver arrives at destination at time $x$, in order to pay a total cost $c_{i}$ he must depart at time $\Lambda_{i}(x)$. The assumption (A2) implies that the maps $\Lambda_{i}: \mathbb{R} \mapsto \mathbb{R}$ are well defined, locally Lipschitz continuous, and nondecreasing.

We now consider an auxiliary problem where all drivers have the same starting and arrival costs, namely

$$
\varphi(x) \doteq-x, \quad \psi(x) \doteq \min _{i=1, \ldots, n} \Lambda_{i}(x) .
$$

Lemma 3.1. Let the flux function $f$ satisfy (A1), and assume that each pair of cost functions $\left(\varphi_{i}, \psi_{i}\right)$, $i=1, \ldots, n$ satisfies (A2). Then the Hamilton-Jacobi equation (2.4) has a unique Nash equilibrium solution corresponding to the cost functions $\varphi, \psi$ in (3.2), where all drivers pay zero total cost.

Proof. In the case all drivers have the same starting and arrival costs, under the assumptions (A1)-(A2) the existence and uniqueness of the Nash equilibrium solution were established by Theorem 3 in [2]. We remark that the functions $\varphi, \psi$ in (3.2) may not satisfy all the assumptions stated in (A2). However, all the arguments used in the original proof in [2] remain valid. Indeed,

- since every map $x \mapsto \Lambda_{i}(x)$ is locally Lipschitz continuous and nondecreasing, the same is true for the map $\psi$ in $(3.2)$;

- since the Nash equilibrium is not affected if we add a constant to the cost functions, in the proof of the existence theorem, the condition $\psi \geq 0$ can be easily replaced by the assumption $\psi$ is bounded below.

To establish a lower bound on $\psi=\min _{i}\left\{\Lambda_{i}(x)\right\}$ we proceed as follows. If $\left.\Lambda_{i}(x)\right) \leq 0$, from the inequalities

$$
c_{i} \geq c_{i}-\psi_{i}(x)=\varphi_{i}\left(\Lambda_{i}(x)\right) \geq \varphi_{i}(0)-\delta_{0} \cdot \Lambda_{i}(x)
$$

we deduce

$$
\Lambda_{i}(x) \geq \frac{\varphi_{i}(0)-c_{i}}{\delta_{0}} \doteq a_{i}
$$

Therefore,

$$
\psi(x) \geq a \doteq \min \left\{a_{1}, \ldots, a_{n}\right\}
$$

- in the proof of Theorem 3, the assumption $\lim _{x \rightarrow+\infty}(\varphi(x)+\psi(x))=+\infty$ is only used to show that all departures must occur within some a-priori bounded interval of time. Therefore, all the arguments in the proof remain valid if we can show that there exists an interval $[a, b]$ such that

$$
x \notin[a, b] \quad \Longrightarrow \quad \psi(x)>x .
$$

If all couples $\left(\varphi_{i}, \psi_{i}\right)$ satisfy the assumptions (A2), we can choose $a$ as in (3.4). We then choose $b>a$ large enough so that $\varphi_{i}(x)+\psi_{i}(x) \geq c_{i}$ for all $x>b$. This yields (3.5).

We recall that initial data for the Nash equilibrium solution admits the representation

$$
Q^{*}(x)=\sup \left\{Q(x) ; \quad Q \in \mathcal{Q}_{0}\right\},
$$


where $\mathcal{Q}_{0}$ is the family of all nondecreasing maps $Q(\cdot)$ as in $(2.6)$, such that the corresponding solution $U$ in (2.8), (2.11) satisfies

$$
\varphi\left(x^{q}(\beta)\right)+\psi\left(x^{a}(\beta)\right) \leq 0 \quad \text { for a.e. } \beta \in[0, Q(+\infty)] .
$$

Otherwise stated, $Q$ should be the initial data for a solution $U$ of $(2.4)$ where a.e. driver has a total cost $\leq 0$.

Next, let $U=U(t, x)$ be the unique Nash equilibrium solution of (2.4) with costs (3.2). We will show that every Nash equilibrium solution of the problem for several groups of drivers with $\operatorname{costs} \varphi_{i}, \psi_{i}$ can be obtained as follows. Consider the unit simplex

$$
\Delta \doteq\left\{\left(\theta_{1}, \ldots, \theta_{n}\right) ; \quad \sum_{i=1}^{n} \theta_{i}=1, \quad \theta_{i} \geq 0 \text { for all } i\right\} .
$$

Let $\Theta$ be the family of all measurable maps

$$
x \mapsto \theta(x)=\left(\theta_{1}(x), \ldots, \theta_{n}(x)\right) \in \Delta
$$

such that

$$
\theta_{i}(x)=0 \quad \text { whenever } \quad \Lambda_{i}(x)>\min _{j=1, \ldots, n} \Lambda_{j}(x) .
$$

Let $\kappa \doteq U(T,+\infty)$. For each map $\theta \in \Theta$, define the prioritizing functions $\mathcal{B}_{i}^{\theta}(\cdot)$ as follows. For all but countably many $\beta \in[0, \kappa]$, there exists a unique arrival time $x^{a}=x^{a}(\beta)$ such that $U\left(T, x^{a}\right)=\beta$. We then set

$$
\mathcal{B}_{i}^{\theta}(\beta) \doteq \int_{-\infty}^{x^{a}(\beta)} \theta_{i}(x) U_{x}(T, x) \mathrm{d} x
$$

Since all functions $\mathcal{B}_{i}^{\theta}$ are Lipschitz continuous with Lipschitz constant 1, they can be uniquely extended to the entire interval $[0, \kappa]$ by continuity. It can be easily checked that the functions $\mathcal{B}_{i}^{\theta}$ are nondecreasing and satisfy (2.21). We then define

$$
\kappa_{i}^{\theta} \doteq \mathcal{B}_{i}^{\theta}(\kappa), \quad Q_{i}^{\theta}(x) \doteq \mathcal{B}_{i}^{\theta}(Q(x)), \quad U_{i}^{\theta}(t, x) \doteq \mathcal{B}_{i}^{\theta}(U(t, x)) .
$$

This clearly yields

$$
\sum_{i=1}^{n} Q_{i}^{\theta}(x)=\sum_{i=1}^{n} \mathcal{B}_{i}^{\theta}(Q(x))=Q(x), \quad \sum_{i=1}^{n} U_{i}^{\theta}(t, x)=\sum_{i=1}^{n} \mathcal{B}_{i}^{\theta}(U(t, x))=U(t, x) .
$$

Theorem 3.2. Let the flux function $f$ satisfy (A1), and assume that each pair of cost functions $\left(\varphi_{i}, \psi_{i}\right)$, $i=1, \ldots, n$ satisfies (A2). Let $U=U(t, x)$ be the unique Nash solution constructed in Lemma 3.1, with initial data $Q(\cdot)$, and let $\Theta$ be the set of all measurable maps $\theta: \mathbb{R} \mapsto \Delta$ satisfying (3.8). Then the following holds.

(i) For every $\theta \in \Theta$, the initial data $Q(\cdot)$ together with the prioritizing functions $\mathcal{B}_{i}^{\theta}$ defined at (3.9) provides a Nash equilibrium solution to the traffic flow problem with costs $\varphi_{i}, \psi_{i}$;

(ii) if the starting distribution $\widetilde{Q}(\cdot)$ together with the prioritizing functions $\widetilde{\mathcal{B}}_{i}$ provide a Nash equilibrium solution with costs $c=\left(c_{1}, \ldots, c_{n}\right)$, then $\widetilde{Q}=Q$ and there exists $\theta \in \Theta$ such that $\widetilde{\mathcal{B}}_{i}=\mathcal{B}_{i}^{\theta}$ for each $i=1, \ldots, n$.

Proof.

1. To prove (i), fix any $\theta \in \Theta$ and $j \in\{1, \ldots n\}$. Since the functions

$$
x \mapsto U(T, x), \quad \beta \mapsto \mathcal{B}_{j}^{\theta}(\beta), \quad x \mapsto U_{j}^{\theta}(T, x)=\mathcal{B}_{j}^{\theta}(U(T, x))
$$


are all continuous and non-decreasing, for all but countably many $\zeta \in\left[0, \kappa_{j}^{\theta}\right]$ the following holds. The point $x_{j}^{a}(\zeta)$ is uniquely determined by the equation

$$
U_{j}^{\theta}\left(T, x_{j}^{a}\right)=\zeta
$$

moreover there exists a unique value $\beta \in[0, \kappa]$ such that $\zeta=\mathcal{B}_{j}^{\theta}(\beta)$, and the point $x^{a}(\beta)$ is uniquely determined by the equation

$$
U\left(T, x^{a}\right)=\beta .
$$

The above uniqueness properties together yield

$$
x_{j}^{a}(\zeta)=x_{j}^{a}\left(\mathcal{B}_{j}^{\theta}(\beta)\right)=x^{a}(\beta) .
$$

2. We now derive a similar identity for the initial time $x^{q}$ when drivers join the queue. Since the functions $x \mapsto Q(x)$ and $x \mapsto Q_{j}^{\theta}(x)=\mathcal{B}_{j}^{\theta}(Q(x))$ are non-decreasing, for all but countably many $\zeta \in\left[0, \kappa_{j}^{\theta}\right]$ the following holds. The point $x_{j}^{q}(\zeta)$ is uniquely determined by the relations

$$
Q_{j}^{\theta}\left(x_{j}^{q}\right) \leq \zeta \leq Q_{j}^{\theta}\left(x_{j}^{q}+\right),
$$

moreover there exists a unique value $\beta \in[0, \kappa]$ such that $\zeta=\mathcal{B}_{j}^{\theta}(\beta)$, and the point $x^{q}(\beta)$ is uniquely determined by the relations

$$
Q\left(x^{q}\right) \leq \beta \leq Q\left(x^{q}+\right) .
$$

The above uniqueness properties together yield

$$
x_{j}^{q}(\zeta)=x_{j}^{q}\left(\mathcal{B}_{j}^{\theta}(\beta)\right)=x^{q}(\beta) .
$$

3. We claim that, if $\zeta \in\left[0, \kappa_{j}\right]$ is a value for which all the uniqueness assumptions in the previous two steps hold, then

$$
\Lambda_{j}\left(x_{j}^{a}(\zeta)\right)=\min _{i=1, \ldots, n} \Lambda_{i}\left(x_{j}^{a}(\zeta)\right) .
$$

Suppose that, on the contrary, $\Lambda_{j}\left(x_{j}^{a}(\zeta)\right)>\min _{i=1, \ldots, n} \Lambda_{i}\left(x_{j}^{a}(\zeta)\right)$. By continuity there exists $\varepsilon>0$ such that

$$
\Lambda_{j}(y)>\min _{i=1, \ldots, n} \Lambda_{i}(y) \quad \text { for all } y \in I_{\varepsilon} \doteq\left[x_{j}^{a}(\zeta), x_{j}^{a}(\zeta)+\varepsilon\right] .
$$

By (3.8) and (3.9), no driver of the $j$-th group arrives during the time interval $I_{\varepsilon}$. Hence $U_{j}(T, \cdot)$ is constant on this interval. For all $y \in I_{\varepsilon}$ we thus have

$$
\begin{aligned}
U_{j}^{\theta}(T, y)=B_{j}^{\theta}(U(T, y)) & =\int_{-\infty}^{y} \theta_{j}(x) U_{x}(T, x) \mathrm{d} x=\int_{-\infty}^{x_{j}^{a}(\zeta)} \theta_{j}(x) U_{x}(T, x) \mathrm{d} x \\
& =\int_{-\infty}^{x^{a}(\beta)} \theta_{j}(x) U_{x}(T, x) \mathrm{d} x=\mathcal{B}_{j}^{\theta}(\beta)=\zeta .
\end{aligned}
$$

In this case, every point $y \in I_{\varepsilon}$ provides a solution to the equation $U_{j}^{\theta}(T, y)=\zeta$. This contradicts the uniqueness assumption on the solution of (3.10). Hence (3.16) must hold. Since the uniqueness assumptions stated in the previous steps hold for all but countably many values of $\zeta$, we conclude that $(3.16)$ is satisfied for a.e. $\zeta \in\left[0, \kappa_{i}\right]$,

4. From the identities (3.12)-(3.15) it now follows

$$
\varphi\left(x_{j}^{q}(\zeta)\right)+\psi\left(x_{j}^{a}(\zeta)\right)=\varphi\left(x^{q}(\beta)\right)+\psi\left(x^{a}(\beta)\right)=0 .
$$


By $(3.2)$ and (3.16) for a.e. $\zeta \in\left[0, \kappa_{i}\right]$ we have $x_{j}^{q}(\zeta)=\min _{i=1, \ldots, n} \Lambda_{i}\left(x_{j}^{a}(\zeta)\right)=\Lambda_{j}\left(x_{j}^{a}(\zeta)\right)$. Hence

$$
\varphi_{j}\left(x_{j}^{q}(\zeta)\right)+\psi_{j}\left(x_{j}^{a}(\zeta)\right)=\varphi_{j}\left(\Lambda_{j}\left(x_{j}^{a}(\zeta)\right)+\psi_{j}\left(x_{j}^{a}(\zeta)\right)=c_{j} .\right.
$$

This proves (2.27).

5. We now prove (2.28). Assume that, on the contrary, there exists $x \in \mathbb{R}$ and an index $j \in\{1, \ldots, n\}$ such that

$$
\varphi_{j}(x)+\psi_{j}(A(x))<c_{j} .
$$

Since $\varphi_{j}$ is monotone decreasing, this implies $x>\Lambda_{j}(A(x))$. Then

$$
\varphi(x)+\psi(A(x))<\varphi\left(\Lambda_{j}(A(x))\right)+\psi(A(x))=-\Lambda_{j}(A(x))+\min _{i=1, \ldots, n} \Lambda_{i}(A(x)) \leq 0 .
$$

This contradicts to the fact that $U(t, x)$ provides a solution to the Nash equilibrium problem with a single population of drivers and costs $\varphi$ and $\psi$ as in (3.2). Hence (2.28) must hold.

6. In the remaining steps we prove part (ii) of the theorem. Assume that the initial distribution $\widetilde{Q}$ together with the prioritizing function $\widetilde{\mathcal{B}}=\left(\widetilde{\mathcal{B}}_{1}, \ldots, \widetilde{\mathcal{B}}_{n}\right)$ provide a Nash equilibrium solution. We begin by proving that $\widetilde{Q}(\cdot)=Q(\cdot)$. For this purpose, it suffices to prove that $\widetilde{Q}(\cdot)$ satisfies $(2.17)$ and (2.18), with $c=0$ and costs $\varphi, \psi$ given by (3.2). The identity $\widetilde{Q}=Q$ will then follow from the uniqueness of the Nash equilibrium solution for a single group of drivers.

Let $\widetilde{U}=\widetilde{U}(t, x)$ be the solution of (2.4) with initial data $\widetilde{Q}$, and fix an index $j \in\{1, \ldots, n\}$. Since $\widetilde{\mathcal{B}}_{j}$ : $[0, \tilde{\kappa}] \mapsto\left[0, \tilde{\kappa}_{j}\right]$ is non-decreasing, for all except countably many values of $\zeta \in\left[0, \tilde{\kappa}_{j}\right]$ the following holds. There is a unique $\beta \in[0, \tilde{\kappa}]$ such that $\zeta \doteq \widetilde{\mathcal{B}}_{j}(\beta)$. Moreover, the departure and arrival times

$$
\tilde{x}_{j}^{q}(\zeta)=\tilde{x}^{q}(\beta), \quad \tilde{x}_{j}^{a}(\zeta)=\tilde{x}^{a}(\beta)
$$

are uniquely determined by the identities

$$
\widetilde{Q}\left(\tilde{x}^{q}(\beta)\right) \leq \beta \leq \widetilde{Q}\left(\tilde{x}^{q}(\beta)+\right), \quad \widetilde{U}\left(T, \tilde{x}^{a}(\beta)\right)=\beta,
$$

and satisfy

$$
\varphi_{j}\left(\tilde{x}^{q}(\beta)\right)+\psi_{j}\left(\tilde{x}^{a}(\beta)\right)=c_{j} .
$$

Since $\widetilde{Q}, \widetilde{\mathcal{B}}$ yield a Nash equilibrium, by $(2.27)$ and (3.1) it follows that

$$
\widetilde{x}^{q}(\beta)=\tilde{x}_{j}^{q}(\zeta)=\Lambda_{j}\left(\tilde{x}_{j}^{a}(\zeta)\right)=\Lambda_{j}\left(\tilde{x}^{a}(\beta)\right) .
$$

Next, we claim that for this particular value of $\beta$ one has

$$
\Lambda_{j}\left(\tilde{x}^{a}(\beta)\right)=\min _{i=1, \ldots, n} \Lambda_{i}\left(\tilde{x}^{a}(\beta)\right) .
$$

Indeed, assume that there exists $k \in\{1, \ldots, n\}$ with $\Lambda_{k}\left(\widetilde{x}^{a}(\beta)\right)<\Lambda_{j}\left(\widetilde{x}^{a}(\beta)\right)$. Since the cost function $\varphi_{k}$ is continuous and strictly decreasing, choosing a starting time $\Lambda_{k}\left(\tilde{x}^{a}(\beta)\right)<y<\tilde{x}^{q}(\beta)$ we achieve

$$
\varphi_{k}(y)+\psi_{k}\left(\tilde{x}^{a}(\beta)\right)<\varphi_{k}\left(\Lambda_{k}\left(\tilde{x}^{a}(\beta)\right)+\psi_{k}\left(\tilde{x}^{a}(\beta)\right)=c_{k} .\right.
$$

Therefore, the total cost to a $k$-driver starting at time $y$ is

$$
\varphi_{k}(y)+\psi_{k}(A(y)) \leq \varphi_{k}(y)+\psi_{k}\left(\tilde{x}^{a}(\beta)\right)<c_{k} .
$$

This yields a contradiction with (2.28), proving our claim. 
7. We can now prove that the initial distribution $\widetilde{Q}$ yields a Nash solution to the problem with costs $\varphi, \psi$ as in (3.2). Since the maps $\widetilde{\mathcal{B}}_{i}$ are non-decreasing and $\widetilde{\mathcal{B}}_{1}(\beta)+\cdots+\widetilde{\mathcal{B}}_{n}(\beta)=\beta$ for all $\beta \in[0, \tilde{\kappa}]$, for a.e. $\beta$ one can find an index $j$ such that $\zeta=\widetilde{\mathcal{B}}_{j}(\beta)$ satisfies the conditions in the previous step. In this case, we have

$$
\tilde{x}^{q}(\beta)=\tilde{x}_{j}^{q}(\zeta)=\Lambda_{j}\left(\tilde{x}_{j}^{a}(\zeta)\right)=\Lambda_{j}\left(\tilde{x}^{a}(\beta)\right)=\min _{i=1, \ldots, n} \Lambda_{i}\left(\tilde{x}^{a}(\beta)\right)=\psi\left(\tilde{x}^{a}(\beta)\right)
$$

Recalling that $\varphi(x)=-x$, for a.e. $\beta \in[0, \tilde{\kappa}]$ we thus have

$$
\varphi\left(\tilde{x}^{q}(\beta)\right)+\psi\left(\tilde{x}^{a}(\beta)\right)=0 .
$$

Hence (2.17) is satisfied.

Finally, to show (2.18), assume by contradiction that there exists $x \in \mathbb{R}$ such that $\varphi(x)+\psi(A(x))<0$. By the definition (3.2), this implies

$$
\min _{i=1, \ldots, n} \Lambda_{i}(A(x))<x
$$

Choose an index $j$ such that $\Lambda_{j}(A(x))=\min _{i=1, \ldots, n} \Lambda_{i}(A(x))$. Since $\varphi_{j}$ is strictly decreasing, one has

$$
\varphi_{j}(x)+\psi_{j}(A(x))<\varphi_{j}\left(\Lambda_{j}(A(x))\right)+\psi_{j}(A(x))=c_{j}
$$

against the assumption (2.28).

This completes the proof that $\widetilde{Q}$ yields a Nash solution, in connection with the cost functions $\varphi, \psi$ in $(3.2)$. By the uniqueness of the Nash solution, we conclude that $\widetilde{Q}(\cdot)=Q(\cdot)$.

8. Thanks to the two previous steps, we can now write $Q, U$ and $\kappa$ in place of $\widetilde{Q}, \widetilde{U}$ and $\tilde{\kappa}$. Let $J \subseteq[0, \kappa]$ be the set of all $\beta \in[0, \kappa]$ such that (i) for every $i=1, \ldots, n$, the differential $\widetilde{\mathcal{B}}_{i}^{\prime}(\beta)=\mathrm{d} \widetilde{\mathcal{B}}_{i} / \mathrm{d} \beta$ at the point $\beta$ is well defined, and (ii) the arrival time $x^{a}(\beta)$ of the $\beta$-driver is uniquely determined by the equation $U\left(T, x^{a}\right)=\beta$.

We observe that meas $([0, \kappa] \backslash J)=0$. Moreover, introducing the set of arrival times

$$
X(J) \doteq\left\{x^{a}(\beta) ; \beta \in J\right\}
$$

we have

$$
\int_{x \notin X(J)} U_{x}(T, x) \mathrm{d} x=0 .
$$

We now define the measurable function $\theta=\left(\theta_{1}, \ldots, \theta_{n}\right):[0, \kappa] \mapsto \Delta_{n}$ by setting

$$
\theta_{i}(x) \doteq \widetilde{\mathcal{B}}_{i}^{\prime}(U(T, x)) \quad \text { if } \quad \beta=U(T, x) \in J,
$$

and choosing $\theta(x) \in \Delta_{n}$ arbitrarily, subject only to the condition (3.8), when $x \notin X(J)$. For all $i \in\{1, \ldots, n\}$ and every $\beta \in J$ using (3.20) we obtain

$$
\begin{aligned}
\mathcal{B}_{i}^{\theta}(\beta) & \doteq \int_{-\infty}^{x^{a}(\beta)} \theta_{i}(x) U_{x}(T, x) \mathrm{d} x=\int_{-\infty}^{x^{a}(\beta)} \widetilde{\mathcal{B}}_{i}^{\prime}(U(T, x)) U_{x}(T, x) \mathrm{d} x \\
& =\int_{0}^{U\left(T, x^{a}(\beta)\right)} \widetilde{\mathcal{B}}_{i}^{\prime}(s) \mathrm{d} s=\int_{0}^{\beta} \widetilde{\mathcal{B}}_{i}^{\prime}(s) \mathrm{d} s=\widetilde{\mathcal{B}}_{i}(\beta) .
\end{aligned}
$$

Since $J$ is dense in $[0, \kappa]$, by continuity the identity $\widetilde{\mathcal{B}}_{i}(\beta)=\mathcal{B}_{i}^{\theta}(\beta)$ remains valid for all $\beta \in[0, \tilde{\kappa}]$. 


\section{NASh EQUilibria With GIVEn POPUlation SIZES}

Assume that $Q(\cdot)$ together with the prioritizing function $\mathcal{B}=\left(\mathcal{B}_{1}, \ldots, \mathcal{B}_{n}\right)$ yields a Nash equilibrium. Let $\kappa \doteq Q(+\infty)$ be the total number of drivers. The size of the $i$-th group of drivers is then $\kappa_{i}=\mathcal{B}_{i}(\kappa)$

For a given vector $c \in \mathbb{R}^{n}$, let $K(c) \subset \mathbb{R}^{n}$ denote the set of all $n$-tuples $\left(\kappa_{1}, \ldots, \kappa_{n}\right)$ which correspond to some Nash solution with costs $c=\left(c_{1}, \ldots, c_{n}\right)$. Our eventual goal is to construct a Nash equilibrium solution for a given $n$-tuple $\left(\kappa_{1}, \ldots, \kappa_{n}\right)$ describing the sizes of the various groups of drivers. This will be achieved by showing that the multifunction $c \mapsto K(c)$ is onto, i.e.

$$
\bigcup_{c \in \mathbb{R}^{n}} K(c)=\mathbb{R}_{+}^{n} \doteq\left\{\left(\kappa_{1}, \ldots, \kappa_{n}\right) ; \quad \kappa_{i} \geq 0 \text { for all } i\right\} .
$$

The next lemma provides a key step in this direction.

Lemma 4.1. The multifunction $c \mapsto K(c)$ is upper semicontinuous, with compact convex values.

Proof.

1. We first show that the multifunction has compact convex values. Fix $c=\left(c_{1}, \ldots, c_{n}\right)$ and let $U(t, x)$ be the unique Nash solution with costs $\varphi, \psi$ as in (3.2), constructed in Lemma 3.1. By Theorem 3.2, we have the following characterization:

$$
K(c)=\left\{\left(\kappa_{1}, \ldots, \kappa_{n}\right) ; \quad \kappa_{i}=\int_{-\infty}^{+\infty} \theta_{i}(x) U_{x}(T, x) \mathrm{d} x, \quad\left(\theta_{1}, \ldots, \theta_{n}\right) \in \Theta\right\} .
$$

By Oleinik's inequality, we have $0 \leq U_{x}(T, x) \leq L$ for some constant $L$ and all $x \in \mathbb{R}$. Therefore $U_{x}(T, \cdot) \in \mathbf{L}^{\infty}$ and the map $\mathcal{K}:\left(\theta_{1}, \ldots, \theta_{n}\right) \mapsto\left(\kappa_{1}, \ldots, \kappa_{n}\right)$ defined by the integral in (4.1) is a continuous linear operator from $\mathbf{L}^{1}\left(\mathbb{R} ; \mathbb{R}^{n}\right)$ into $\mathbb{R}^{n}$. The operator $\mathcal{K}$ is trivially compact, because the range is finite dimensional. Since the domain $\Theta$ defined at (3.8) is a closed convex, bounded subset of $\mathbf{L}^{1}\left(\mathbb{R} ; \mathbb{R}^{n}\right)$, we conclude that its image $K(c)=\mathcal{K}(\Theta)$ is a compact, convex subset of $\mathbb{R}^{n}$.

2. To prove that the map $c \mapsto K(c)$ is upper semicontinuous, i.e. it has closed graph, consider a sequence of Nash equilibrium solutions corresponding to $\operatorname{costs} c^{\nu}=\left(c_{1}^{\nu}, \ldots, c_{n}^{\nu}\right), \nu \geq 1$. According to Definition 2.3, these solutions are determined by an initial data $Q^{\nu}(\cdot)$ together with the prioritizing functions: $\mathcal{B}_{i}^{\nu}:\left[0, \kappa^{\nu}\right] \mapsto\left[0, \kappa_{i}^{\nu}\right]$, with $\kappa^{\nu}=Q^{\nu}(+\infty)$, so that the conditions (2.27)-(2.28) hold.

Assume that, for every $i=1, \ldots, n$,

$$
c_{i}^{\nu} \rightarrow \bar{c}_{i}, \quad \kappa_{i}^{\nu} \rightarrow \bar{\kappa}_{i} \quad \text { as } \quad \nu \rightarrow \infty .
$$

Of course, this implies

$$
\kappa^{\nu}=\sum_{i=1}^{n} \kappa_{i}^{\nu} \rightarrow \sum_{i=1}^{n} \bar{\kappa}_{i}=\bar{\kappa} \quad \text { as } \quad \nu \rightarrow \infty .
$$

We need to show that there exists a Nash equilibrium solution with $\operatorname{costs} \bar{c}=\left(\bar{c}_{1}, \ldots, \bar{c}_{n}\right)$ and total population sizes $\left(\bar{\kappa}_{1}, \ldots, \bar{\kappa}_{n}\right)$.

By (2.21), all maps $\mathcal{B}_{i}^{\nu}:\left[0, \kappa^{\nu}\right] \mapsto \mathbb{R}$ are Lipschitz continuous with constant 1 . Therefore, by possibly taking a subsequence, we can achieve the pointwise convergence $\mathcal{B}_{i}^{\nu}(\beta) \rightarrow \overline{\mathcal{B}}_{i}(\beta)$ for all $\beta \in[0, \bar{\kappa}$. This convergence is uniform on every compact subinterval. By possibly taking a further subsequence, by Helly's compactness theorem we can assume that $Q^{\nu}(x) \rightarrow \bar{Q}(x)$ pontwise, for every $x \in \mathbb{R}$. Clearly, $\bar{Q}(+\infty)=\bar{\kappa}$.

Next, for every $i \in\{1, \ldots, n\}$ and $\nu \geq 1$, recall $\kappa_{i}^{\nu}=Q_{i}^{\nu}(+\infty)$ and consider the functions $\beta_{i}^{\nu}:\left[0, \kappa_{i}^{\nu}\right] \mapsto\left[0, \kappa^{\nu}\right]$ defined as

$$
\beta_{i}^{\nu}(\zeta)=\min \left\{\beta ; \mathcal{B}_{i}^{\nu}(\beta)=\zeta\right\}
$$


Observe that this construction yields

$$
\mathcal{B}_{i}^{\nu}\left(\beta_{i}^{\nu}(\zeta)\right)=\zeta \quad \text { for all } \zeta \in\left[0, \kappa_{i}^{\nu}\right] .
$$

With obvious meaning of notation, we also consider the departure and arrival times

$$
x_{i}^{q, \nu}(\zeta)=x^{q, \nu}\left(\beta_{i}^{\nu}(\zeta)\right), \quad x_{i}^{a, \nu}(\zeta)=x^{a, \nu}\left(\beta_{i}^{\nu}(\zeta)\right) .
$$

Since all these functions are bounded and monotone, by possibly taking a further subsequence we can assume the pointwise convergence

$$
\begin{aligned}
\beta_{i}^{\nu}(\zeta) \rightarrow \bar{\beta}_{i}(\zeta) & \text { for all } \zeta \in\left[0, \bar{\kappa}_{i}[,\right. \\
x_{i}^{q, \nu}(\zeta) \rightarrow \bar{x}_{i}^{q}(\zeta)=\bar{x}^{q}\left(\bar{\beta}_{i}(\zeta)\right), & x_{i}^{a, \nu}(\zeta) \rightarrow \bar{x}_{i}^{a}(\zeta)=\bar{x}^{a}\left(\bar{\beta}_{i}(\zeta)\right),
\end{aligned}
$$

with

$$
\overline{\mathcal{B}}_{i}\left(\bar{\beta}_{i}(\zeta)\right)=\zeta \quad \text { for a.e. } \zeta \in\left[0, \bar{\kappa}_{i}\right] .
$$

From the identities

$$
\varphi_{i}\left(x_{i}^{q, \nu}(\zeta)\right)+\psi_{i}\left(x_{i}^{a, \nu}(\zeta)\right)=c_{i}^{\nu} \quad \text { for a.e. } \zeta \in\left[0, \kappa_{i}^{\nu}\right],
$$

letting $\nu \rightarrow \infty$ and using the continuity of $\varphi_{i}, \psi_{i}$ we conclude

$$
\varphi_{i}\left(\bar{x}_{i}^{q}(\zeta)\right)+\psi_{i}\left(\bar{x}_{i}^{a}(\zeta)\right)=\bar{c}_{i} \quad \text { for a.e. } \zeta \in\left[0, \bar{\kappa}_{i}\right] .
$$

Hence (2.27) holds.

To complete the proof, it remains to show that

$$
\varphi_{i}(x)+\psi_{i}(\bar{A}(x)) \geq \bar{c}_{i} \quad \text { for all } x \in \mathbb{R},
$$

where $\bar{A}$ is the arrival map corresponding to the departure distribution $\bar{Q}$ :

$$
\bar{A}(x) \doteq \max \left\{x+\mu, \sup _{\beta<\bar{Q}(x)} \bar{x}^{a}(\beta)\right\} .
$$

If (4.2) fails, by continuity we can find $y \in \mathbb{R}$ and $\delta>0$ such that

$$
\varphi_{i}(y-\delta)+\psi_{i}(\bar{A}(y)) \leq \bar{c}_{i}-\delta
$$

We now consider the arrival maps

$$
A^{\nu}(x) \doteq \max \left\{x+\mu, \sup _{\beta<Q^{\nu}(x)} x^{a, \nu}(\beta)\right\}
$$

and observe that

$$
\limsup _{\nu \rightarrow \infty} A^{\nu}(y-\delta) \leq \bar{A}(y)
$$

From the inequalities

$$
\varphi_{i}(y-\delta)+\psi_{i}\left(A^{\nu}(y-\delta)\right) \geq c_{i}^{\nu},
$$

letting $\nu \rightarrow \infty$ and using (4.6), since $\varphi_{i}^{\prime} \leq 0$ while $\psi_{i}^{\prime} \geq 0$, we conclude

$$
\varphi_{i}(y-\delta)+\psi_{i}(\bar{A}(y)) \geq \bar{c}_{i} .
$$

This yields a contradiction with (4.4), completing the proof. 
In order to prove the existence of Nash equilibrium solutions to the traffic problem with given population sizes $\bar{\kappa}=\left(\bar{\kappa}_{1}, \ldots, \bar{\kappa}_{n}\right)$, we shall use a topological argument, based on Bruwer's fix point theorem.

In the following, we denote by $\left\{\mathbf{e}_{1}, \ldots, \mathbf{e}_{n}\right\}$ the standard basis in $\mathbb{R}^{n}$, and let

$$
\Delta \doteq\left\{x \in \mathbb{R}^{n} ; \quad, \quad \sum_{i=1}^{n} \mathbf{e}_{i} \cdot x \leq 1, \quad \mathbf{e}_{i} \cdot x \geq 0 \quad \text { for all } i=1, \ldots, n\right\}
$$

be the unit simplex in $\mathbb{R}^{n}$. Moreover, we consider the vector

$$
\overline{\mathbf{e}} \doteq \sum_{i=1}^{n} \mathbf{e}_{i}=(1,1, \ldots, 1) \in \mathbb{R}^{n} .
$$

Lemma 4.2. The multifunction $c \mapsto K(c)$ satisfies the following properties.

(i) If $\left(\kappa_{1}, \ldots, \kappa_{n}\right) \in K\left(c_{1}, \ldots, c_{n}\right)$ and one of the costs satisfies

$$
c_{j} \leq \gamma_{0} \doteq \min _{i=1, \ldots, n} \min _{x \in \mathbb{R}}\left\{\varphi_{i}(x)+\psi_{i}(x)\right\}
$$

then $\kappa_{j}=0$;

(ii) For every $M>0$, there exists a constant $C_{M}$ such that:

$$
\text { if } \sum_{i=1}^{n} c_{i} \geq C_{M} \quad \text { and } \quad\left(\kappa_{1}, \ldots, \kappa_{n}\right) \in K\left(c_{1}, \ldots, c_{n}\right), \quad \text { then } \quad \sum_{i=1}^{n} \kappa_{i} \geq M \text {. }
$$

Proof.

( $i$ ) In view of assumption (A2), $\gamma_{0}$ is well defined real number. Since any driver starting at time $x$ must arrive at some time $A(x)>x$, the total cost to a driver of the $j$-th group satisfies the inequality

$$
\varphi_{j}(x)+\psi_{j}(A(x))>\varphi_{j}(A(x))+\psi_{j}(A(x)) \geq \gamma_{0} \geq c_{j}
$$

Since no $j$-driver can achieve a cost $\leq c_{j}$, the total number of $j$-drivers must be zero.

(ii) Let $\tilde{u}$ be any solution to the conservation law (2.1) with total mass $\int \tilde{u}(t, x) \mathrm{d} x=M$ and having compact support, say, contained in the domain $[0, T] \times[a, b]$. We claim that (4.10) is satisfied by choosing the constant $C_{M} \geq 0$ large enough so that

$$
C_{M} \geq n \cdot \max _{i=1, \ldots, n}\left\{\varphi_{i}(a)+\psi_{i}(b)\right\} .
$$

Indeed, assume that the vector of $\operatorname{costs} c=\left(c_{1}, \ldots, c_{n}\right)$ satisfies $\sum c_{i} \geq C_{M}$, and let $\left(\kappa_{1}, \ldots, \kappa_{n}\right) \in K(c)$. Consider the functions $\Lambda_{i}(\cdot)$ as in (3.1). Observe that the choice of the constant $C_{M}$ in (4.11) yields

$$
\min _{i=1, \ldots, n} \Lambda_{i}(x) \leq \min _{i=1, \ldots, n} \Lambda_{i}(b) \leq a \quad \text { for all } x \in[a, b] .
$$

In view of Theorem 3.2, it suffices to prove that the Nash solution $U^{*}$, for a single group of drivers with cost functions $\varphi, \psi$ as in (3.2) and total cost zero, contains a total number of drivers $\geq M$.

We observe that, in the above solution with density $\tilde{u}(t, x)$, every driver departs and arrives at some times $x^{q}, x^{a} \in[a, b]$, and pays a total cost

$$
\varphi\left(x^{q}\right)+\psi\left(x^{a}\right)=-x^{q}+\min _{i=1, \ldots, n} \Lambda_{i}\left(x^{a}\right) \leq-x^{q}+a \leq 0 .
$$




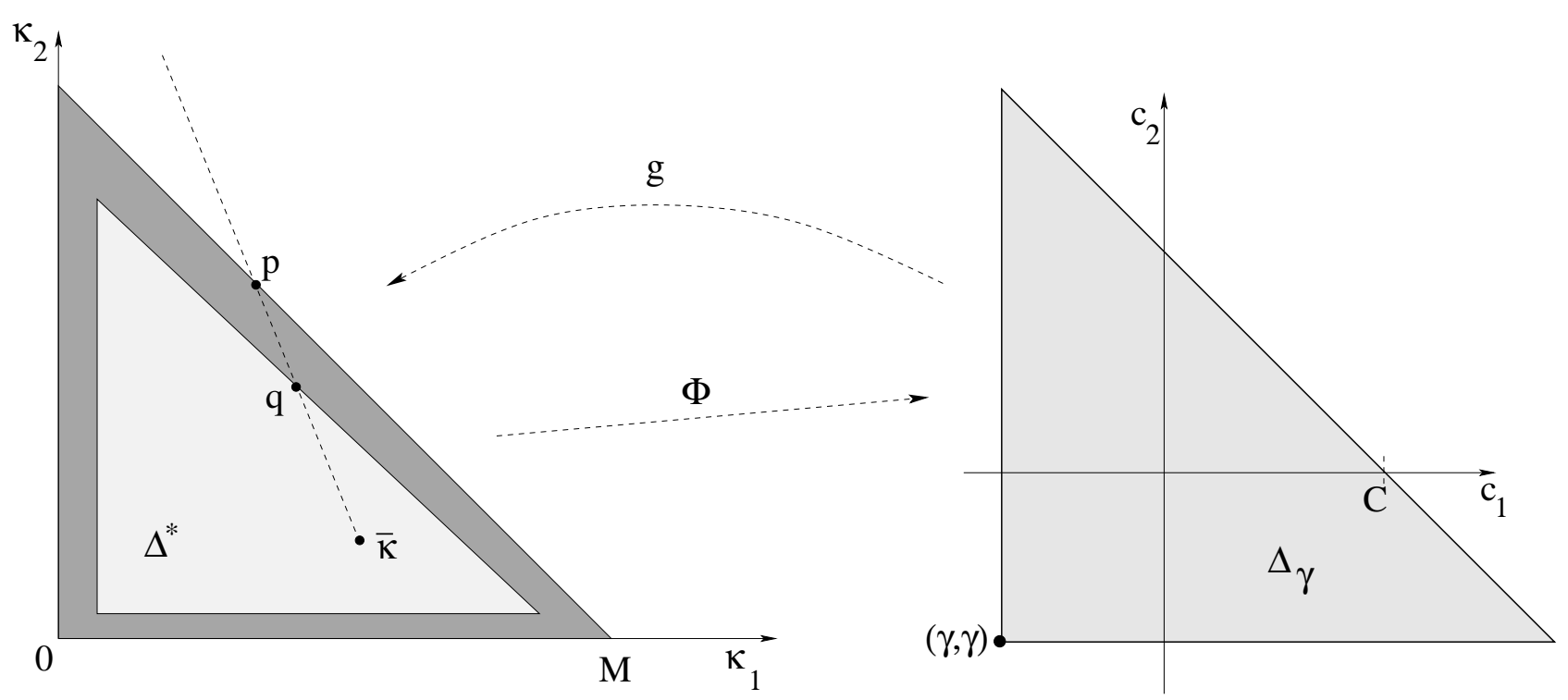

Figure 2. The simplexes $\Delta^{*}=\Delta^{\text {inner }} \cup \Delta^{\text {outer }}$, and $\Delta_{\gamma}$.

Therefore, the integral function $\widetilde{U}(0, x) \doteq \int_{-\infty}^{x} \tilde{u}(0, y) \mathrm{d} y$ is contained in the family $\mathcal{Q}_{0}$ of all departure distributions where each driver bears a total cost $\leq 0$.

Calling $Q^{*}(\cdot)$ the initial data for the Nash solution, the representation (3.6) now yields

$$
\kappa_{1}+\cdots+\kappa_{n}=Q^{*}(+\infty) \geq U(0,+\infty)=M .
$$

\subsection{Proof of Theorem 2.4}

With the aid of the previous lemmas, we can now give a proof of Theorem 2.4. Clearly, it suffices to prove that the upper semicontinuous, compact convex valued multifunction $c \mapsto K(c) \subset \mathbb{R}_{+}^{n}$ is surjective, namely

$$
\bigcup_{c \in \mathbb{R}^{n}} K(c)=\mathbb{R}_{+}^{n}
$$

We shall argue by contradiction. Assume that, for some vector $\bar{\kappa}=\left(\bar{\kappa}_{1}, \ldots, \bar{\kappa}_{n}\right) \in \mathbb{R}_{+}^{n}$ is not contained in the left hand side of (4.13). It is not restrictive to assume that $\bar{\kappa}_{i}>0$ for every $i$. Indeed, in the general case we can simply take $c_{j}=\gamma_{0}$ for every index $j$ such that $\bar{\kappa}_{j}=0$, and consider a lower dimensional problem.

1. Using Lemma 4.2, fix any $M>\sum_{i} \bar{\kappa}_{i}$ and choose $C_{M}$ so that (4.10) holds. We then choose $\gamma<\gamma_{0}$ and $C>C_{M}-\gamma_{0}$. Consider the two $n$-dimensional simplexes (see Fig. 2)

$$
\begin{aligned}
\Delta_{\gamma} \doteq\left\{\left(c_{1}, \ldots, c_{n}\right) ; \quad \sum_{i=1}^{n} c_{i} \leq C, \quad c_{i} \geq \gamma \quad \text { for all } i\right\}, \\
\Delta^{*} \doteq\left\{\left(\kappa_{1}, \ldots, \kappa_{n}\right) ; \quad \sum_{i=1}^{n} \kappa_{i} \leq M, \quad \kappa_{i} \geq 0 \quad \text { for all } i\right\} .
\end{aligned}
$$


Let $\Phi=\left(\Phi_{1}, \ldots, \Phi_{n}\right): \Delta^{*} \mapsto \Delta_{\gamma}$ be the natural affine bijection between these two simplexes:

$$
\Phi_{i}\left(\kappa_{1}, \ldots, \kappa_{n}\right) \doteq \gamma+\frac{C-n \gamma}{M} \kappa_{i}
$$

2. By assumption,

$$
\bar{\kappa} \notin \bigcup_{c \in \Delta_{\gamma}} K(c) .
$$

Since the multifunction $K$ is upper semicontinuous and $\Delta_{\gamma}$ is compact, we can find $r>0$ such that the ball $B(\bar{\kappa}, r)$ does not intersect the right hand side of (4.17).

3. By Cellina's approximate selection theorem [3], for any $\delta>0$ there exists a continuous map $g=$ $\left(g_{1}, \ldots, g_{n}\right): \Delta_{\gamma} \mapsto \mathbb{R}_{+}^{n}$ such that

$$
\operatorname{Graph}(g) \subset B(\operatorname{Graph}(K), \delta)
$$

For any $\varepsilon>0$, by choosing $\delta>0$ small enough we can achieve

$$
\begin{gathered}
c_{i}=\gamma \quad \Longrightarrow \quad g_{i}(c)<\varepsilon, \\
\sum_{i=1}^{n} c_{i}=C \quad \Longrightarrow \quad \sum_{i=1}^{n} g_{i}(c)>M-\varepsilon, \\
|g(c)-\bar{\kappa}|>\frac{3}{4} r
\end{gathered}
$$

for all $c \in \Delta_{\gamma}$.

4. Consider the composed map $g \circ \Phi: \Delta^{*} \mapsto \mathbb{R}^{n}$. We claim that, choosing $\eta>0$ sufficiently small, the following holds. For every boundary point $p \in \partial \Delta^{*}$, define the nearby point

$$
q=\eta \bar{\kappa}+(1-\eta) p
$$

Then the segment

$$
S_{p} \doteq\{\theta(g \circ \Phi)(q)+(1-\theta) p ; \quad \theta \in[0,1]\}
$$

with endpoints $p$ and $(g \circ \Phi)(q)$ does not intersect the ball $B(\bar{\kappa}, r / 2)$.

Indeed, given $p=\left(p_{1}, \ldots, p_{n}\right) \in \partial \Delta^{*}$, two cases can arise.

Case 1. $\sum_{i} p_{i}=M$. In this case $\sum_{i} \Phi_{i}(p)=C$, hence the components of $g=\left(g_{1}, \ldots, g_{n}\right)$ satisfy $\sum_{i} g_{i} \circ \Phi(p) \geq M-\varepsilon$. Therefore, the segment $S_{p}$ cannot intersect the ball $B(\bar{\kappa}, r / 2)$ because

$$
S_{p} \subset\left\{\left(\kappa_{1}, \ldots, \kappa_{n}\right) ; \quad \sum_{i} \kappa_{i} \geq M-\varepsilon\right\} .
$$

Case 2. There exists an index $j \in\{1, \ldots, n\}$ such that $p_{j}=0$. In this case, $\Phi_{j}(p)=\gamma$ and hence $g_{j} \circ \Phi(p)<\varepsilon$. Therefore, the segment $S_{p}$ cannot intersect the ball $B(\bar{\kappa}, r / 2)$ because

$$
S_{p} \subset\left\{\left(\kappa_{1}, \ldots, \kappa_{n}\right) ; \quad \kappa_{j} \leq \varepsilon\right\} .
$$


5. Consider the decomposition $\Delta^{*}=\Delta^{\text {inner }} \cup \Delta^{\text {outer }}$ into an inner and an outer domain, defined as

$$
\begin{aligned}
& \left.\left.\Delta^{\text {inner }} \doteq\left\{x \in \Delta^{*} ; x=\theta \bar{\kappa}+(1-\theta) p \quad \text { for some } p \in \partial \Delta^{*}, \theta \in\right] \eta, 1\right]\right\}, \\
& \Delta^{\text {outer }} \doteq\left\{x \in \Delta^{*} ; x=\theta \bar{\kappa}+(1-\theta) p \quad \text { for some } p \in \partial \Delta^{*}, \theta \in[0, \eta]\right\} .
\end{aligned}
$$

We now consider the continuous map $h: \Delta^{*} \mapsto \mathbb{R}^{n}$, defined as follows.

- If $\kappa \in \Delta^{\text {inner }}$, we define $h(\kappa) \doteq g \circ \Phi(\kappa)$;

- if $\kappa \in \Delta^{\text {outer }}$, then there exists a unique point $p \in \partial \Delta^{*}$ and a unique value $\theta \in[0,1]$ such that

$$
\kappa=\theta p+(1-\theta) q \quad q=\eta \bar{\kappa}+(1-\eta) p .
$$

In this case, we define

$$
h(\kappa) \doteq \theta p+(1-\theta)(g \circ \Phi)(q) .
$$

6. Let $\pi^{*}$ the radial projection onto $\partial \Delta^{*}$ along rays centered at $\bar{\kappa}$. More precisely, for $x \neq \bar{\kappa}$, define $\pi^{*}(x)$ as the unique intersection of the ray $\{\bar{\kappa}+\lambda(x-\bar{\kappa}) ; \quad \lambda>0\}$ with the boundary $\partial \Delta^{*}$.

By the analysis in step 4, the image $h\left(\Delta^{*}\right)$ does not intersect the ball $B(\bar{\kappa}, r / 2)$. Therefore, the composition $\kappa \mapsto \pi^{*} \circ h(\kappa)$ is a continuous map from the compact simplex $\Delta^{*}$ onto its boundary, such that

$$
\pi^{*} \circ h(\kappa)=\kappa \quad \text { for all } \kappa \in \partial \Delta^{*} .
$$

According to Brouwer's theorem, such a continuous retraction does not exist. This contradiction proves our theorem

Example 4.3. To see that the map $c \mapsto \kappa(c)$ can be multivalued, a trivial case is the following. Consider a pair of cost functions $\varphi, \psi$ satisfying the assumptions (A2). Choose any cost $c_{0} \in \mathbb{R}$ such that the problem with a single group of drivers has a solution $Q(\cdot)$ with total mass $\bar{\kappa}$, and common cost $c_{0}$. Now consider the problem with $n$ groups of drivers, where all groups have the same starting and arrival cost $\varphi_{i}=\varphi, \psi_{i}=\psi$ for all $i=1, \ldots, n$. Then $Q(\cdot)$ together with a completely arbitrary prioritizing function yields a Nash equilibrium solution. Hence, if $c=\left(c_{0}, c_{0}, \ldots, c_{0}\right)$, then the set

$$
\kappa(c)=\left\{\left(\kappa_{1}, \ldots, \kappa_{n}\right) ; \quad \kappa_{i} \geq 0, \quad \sum_{j=1}^{n} \kappa_{j}=\bar{\kappa}\right\}
$$

is a compact, convex simplex.

While the map $c \mapsto \kappa(c)$ is generally multivalued, it seems reasonable to expect that a partial uniqueness result should hold for the inverse map $\kappa \mapsto c$. This leads to the following conjecture: If $\kappa_{i}>0$ for every $i \in\{1, \ldots, n\}$, then there exists a unique $n$-tuple $c=\left(c_{1}, \ldots, c_{n}\right)$ such that $\left(\kappa_{1}, \ldots, \kappa_{n}\right) \in \kappa(c)$. In the special case $n=1$, the validity of this conjecture was proved in [2].

\section{REFERENCES}

[1] J.P. Aubin and A. Cellina, Differential inclusions. Set-Valued Maps and Viability Theory. Springer-Verlag, Berlin (1984).

[2] A. Bressan and K. Han, Optima and equilibria for a model of traffic flow. SIAM J. Math. Anal. 43 (2011) $2384-2417$.

[3] A. Cellina, Approximation of set valued functions and fixed point theorems. Ann. Mat. Pura Appl. 82 (1969) 17-24. 
[4] F.H. Clarke, Yu.S. Ledyaev, R.J. Stern and P.R. Wolenski, Nonsmooth Analysis and Control Theory. Springer-Verlag, New York (1998).

[5] T.L. Friesz, Dynamic Optimization and Differential Games, Springer, New York (2010).

[6] T.L. Friesz, T. Kim, C. Kwon and M.A. Rigdon, Approximate network loading and dual-time-scale dynamic user equilibrium. Transp. Res. Part B (2010).

[7] A. Fügenschuh, M. Herty and A. Martin, Combinatorial and continuous models for the optimization of traffic flows on networks. SIAM J. Optim. 16 (2006) 1155-1176.

[8] M. Garavello and B. Piccoli, Traffic Flow on Networks. Conservation Laws Models. AIMS Series on Applied Mathematics, Springfield, Mo. (2006).

[9] M. Gugat, M. Herty, A. Klar and G. Leugering, Optimal control for traffic flow networks. J. Optim. Theory Appl. 126 (2005) $589-616$.

[10] M. Herty, C. Kirchner and A. Klar, Instantaneous control for traffic flow. Math. Methods Appl. Sci. 30 (2007) 153-169.

[11] L.C. Evans, Partial Differential Equations, 2nd edition. American Mathematical Society, Providence, RI (2010).

[12] P.D. Lax, Hyperbolic systems of conservation laws II. Commun. Pure Appl. Math. 10 (1957) 537-566.

[13] M. Lighthill and G. Whitham, On kinematic waves. II. A theory of traffic flow on long crowded roads. Proc. R. Soc. Lond. Ser. A 229 (1955) 317-345.

[14] P.I. Richards, Shock waves on the highway. Oper. Res. 4 (1956), 42-51.

[15] J. Smoller, Shock waves and reaction-diffusion equations, 2nd edition. Springer-Verlag, New York (1994). 